\title{
NEW CONNECTION METHOD ACROSS MORE GENERAL TURNING POINTS ${ }^{1}$
}

\author{
BY J. F. PAINTER AND R. E. MEYER
}

The basic oscillator or Schroedinger equation

$$
\epsilon^{2} d^{2} w / d z^{2}+q^{2} w(z)=0, \quad \epsilon \downarrow 0,
$$

with $q(z)$ analytic, except for a root or singular point at $z=0$, poses a wellknown connection problem. WKBJ or $L-G$ approximations

$$
\begin{aligned}
w & \sim A q^{-1 / 2} \exp (i \xi / \epsilon)+B q^{-1 / 2} \exp (-i \xi / \epsilon), \\
d w / z & \sim\left(i q^{1 / 2} / \epsilon\right)[A \exp (i \xi / \epsilon)-B \exp (-i \xi / \epsilon)]
\end{aligned}
$$

with

$$
\xi=\int_{0}^{z} q(s) d s
$$

and constants $A, B$ are valid in suitable regions, but not in a neighborhood of the transition point. The constants $A_{l}, B_{l}$ in (2) valid to the left of such a point generally differ from those, $A_{r}, B_{r}$, valid to the right. Langer [1] found one constant pair in terms of the other for the class of "fractional turning points" at which $z^{-v} q(z)$ is analytic for real $v>-1$. But, if branch points, even of infinite order, be thus admitted, why not logarithms of $z$ and still other branch points?

The reason lies in his "central connection" method by uniform approximation even near $z=0$. For fractional turning points such approximands to $w(z)$ are furnished by Bessel functions, but for logarithmic turning points they are necessarily less tractable. Uniform approximation, however, is not a prerequisite for connection, nor is it needed for the most important physical applications, e.g., in scattering.

A new method will now be summarized which solves the problem for a more general class of equations (1) characterized (a precise statement is found in the Appendix) by

$$
\varphi(\xi):=1 / 2 q^{-2} d q / d z=\xi^{-1}(\gamma+o(1)) \quad \text { as } \xi \rightarrow 0
$$

with real $\gamma<1 / 2$. This includes the fractional turning points, where $\gamma=1 / 2 v /(1+v)$ $(<1 / 2$ since $v>-1)$, and also logarithmic turning points, where $q(z) \sim z^{v}(\log z)^{\mu}$

Received by the editors June 12, 1980 and, in revised form, November 17, 1980. 1980 Mathematics Subject Classification. Primary 34E20; Secondary 41 A60.

${ }^{1}$ Supported by NSF and WARF, sponsored by the U. S. Army under Contract DAAG29-80-C-0041. 
with real $\mu$ and $\gamma$ has the same value, but the $o(1)$ term in (4) is more disagreeable, and also a great many more general branch points of $q(z)$.

The connection problem has many related versions; for definiteness, the branch cut will be taken along the negative real $\xi$-axis (Figure 1) and connection,

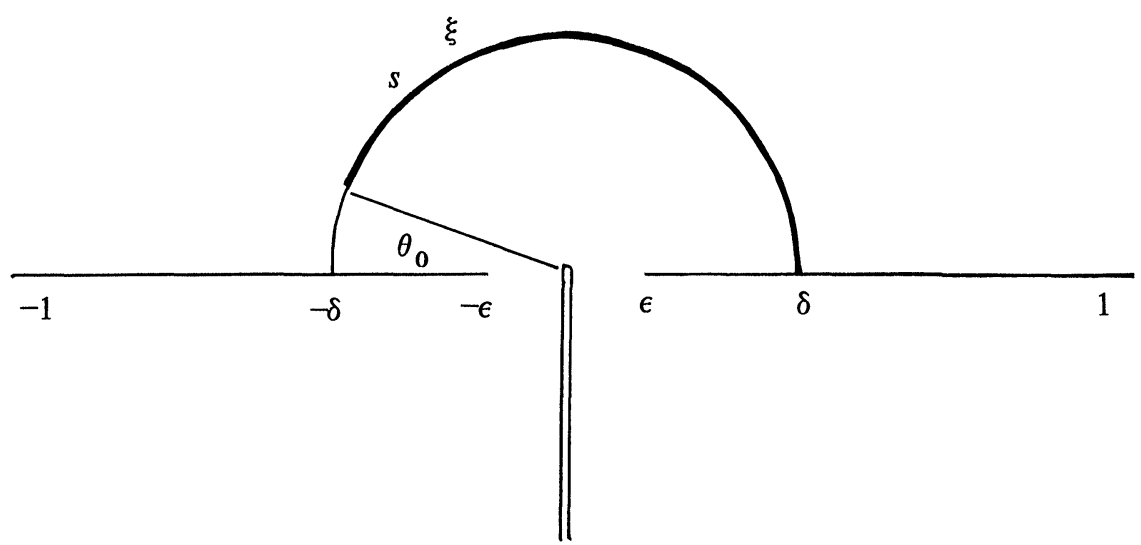

FIGURE 1

attempted from $\xi=-1$ on the left to $\xi=1$ on the right. For the term in (2) dominant above the real axis, a simple asymptotic argument [2] then gives $B_{r}=$ $B_{l}+o(1)$, the difficulty lies in finding $A_{r}$. Let $A(\xi), B(\xi)$ denote the functions for which (2) is an identity, not an asymptotic relation; then (1) can be cast as an integral equation

$$
A(\xi)=A_{l}+B_{l} j\left(\xi, \xi_{l}\right) e^{-\rho \xi_{l}}+\int_{\xi_{l}}^{\xi} A(s) j(\xi, s) \varphi(s) d s
$$

with $\xi_{l}$ any negative number and

$$
\rho=2 i / \epsilon, \quad j(\xi, s)=e^{\rho s} \int_{s}^{\xi} e^{-\rho t} \varphi(t) d t, \quad A_{l}=A\left(\xi_{l}\right), \quad B_{l}=B\left(\xi_{l}\right) .
$$

It is studied first on a semicircle $|\xi|=\delta(\epsilon)$ (Figure 1), so that $\xi_{l}=-\delta, \xi_{r}=\delta$ and

$$
\delta(\epsilon) \rightarrow 0, \quad \epsilon / \delta(\epsilon) \rightarrow 0 \quad \text { as } \epsilon \rightarrow 0 .
$$

This involves a drastic departure from the key requirement of turning point theory [3] to specify integrals only along "progressive paths" along which the exponentials in the integrands vary monotonely in magnitude. In the new method, nonmonotone and wild variation of the exponentials is central, a price paid for gains in flexibility.

By careful choice of the paths of integration, the kernel $j(\xi, s)$ can be approximated [4] for $\xi$ and $s$ on the arc emphasized in Figure 1 (where $\theta_{0}>0$ 
and independent of $\epsilon$ ) by an incomplete gamma function, whence in turn,

$$
j(\xi, s) e^{-\rho s}=[\gamma+o(1)]\left\{e^{-\rho s} / \rho s-e^{-\rho \xi} / \rho \xi\right\}
$$

on that arc. Related estimates for other $\xi, s$ on the semicircle [4] complement (6), and by splitting off a volatile exponential factor, the integral operator in (5) may be made to contract-but only a disappointing bound $|A(\xi)| \leqslant$ const. times $\exp (2 \delta / \epsilon)$ is so obtainable for $\xi$ on the semicircle.

Now, for $\varphi(\xi)=\gamma / \xi$ exactly, because $q(z)=z^{v}$ exactly, (1) is solved in terms of Hankel functions by

$$
w=\xi^{\lambda} H_{\lambda}^{(i)}(\xi / \epsilon), \quad \lambda=1 / 2-\gamma, \quad i=1 \text { or } 2,
$$

whence $A(\xi)$ can be calculated for this special case; call this function $A^{\gamma}(\xi)$. Then (5) can be cast as an integral equation for $A(\xi)-A^{\gamma}(\xi)$, and a contraction argument yields [4] $A(\xi)-A^{\gamma}(\xi) \rightarrow 0$ as $\epsilon \rightarrow 0$ uniformly on the entire semicircle $|\xi|=\delta(\epsilon)$ (Figure 1) provided $\delta(\epsilon)$ be chosen so that

$$
(\epsilon / \delta)[\delta \varphi(\delta)-\gamma] \exp (2 \delta / \epsilon) \rightarrow 0 \quad \text { as } \epsilon \rightarrow 0 .
$$

The known connection formula for (7) is thereby extended to $A$ :

$$
A(\delta(\epsilon))=A(-\delta(\epsilon))-2 i B(-\delta(\epsilon)) \sin (\gamma \pi)+o(1) .
$$

Unfortunately, this requires a $\delta(\epsilon)$ so close to $\epsilon$ that the extension from $\xi=\delta$ to $\xi=1$ (and $-\delta$ to -1 ) necessary for practical usefulness of connection, remains a real problem. For (5) on the real axis, a contraction argument [4] shows $A(\xi)$ to be approximately constant over intervals of sufficiently short length (dependent on $\epsilon$ and $\delta$ ). They are generally too short to reach from $\delta(\epsilon)$ to 1 , but a finite chain of them [4] does yield the desired extension, provided (4) is slightly strengthened to

$$
\xi \varphi(\xi)=\gamma+o(\log \log \ldots \log |\xi|)^{-1} \text { as } \xi \rightarrow 0
$$

which still admits logarithmic turning points and many more. Then

$$
\begin{aligned}
& A(1)=A(-1)-2 i B(-1) \sin (\gamma \pi)+o(1), \\
& B(1)=B(-1)+o(1)
\end{aligned}
$$

as $\epsilon \rightarrow 0$. Much in contrast to the uniform approximation to the solution of (1), the first approximation to connection is thus the same for our generalized turning points as for Langer's fractional ones-and for Bessel functions.

Appendix. A precise statement of the assumptions assuring (9) is that $q(z)$ in (1) is analytic and nonzero on an open, connected and simply connected set $R_{z}$ of complex numbers and also at every point of $\partial R_{z}$, except for $z=0$. At $z=0, q(z)$ is absolutely integrable so that $\xi$ can be defined by (3). The image $R_{\xi}=\xi\left(R_{z}\right)$ contains a fixed rectangular neighborhood of the branch point, except 
for the cut. On $R_{\xi}, \varphi(\xi)$ is of exponential order as $|\xi| \rightarrow-i \infty$, and (8) holds with real constant $\gamma<1 / 2$ and some finite iterate of the logarithm.

\section{REFERENCES}

1. R. E. Langer, On the asymptotic solutions of differential equations, with an application to Bessel functions of large complex order, Trans. Amer. Math. Soc. 34 (1932), 447464.

2. M. A. Evgrafov and M. V. Fedoryuk, Asymptotic behaviour as $\lambda \rightarrow \infty$ of the solution of the equation $w^{\prime \prime}(z)-p(z, \lambda) w(z)=0$ in the complex z-plane, Uspehi Mat. Nauk. 21 (1966), 3-50 = Russian Math. Surveys 21 (1966), $1-48$.

3. F. W. J. Olver, Asymptotics and special functions, Academic Press, New York, 1974.

4. J. F. Painter and R. E. Meyer, Connection at close quarters to generalized turning points, Math. Res. Ctr., Univ. Wis., Tech. Sum. Rep. 2068, 1980.

L-307, LAWRENCE LIVERMORE LABORATORY, LIVERMORE, CALIFORNIA 94550

MATHEMATICS RESEARCH CENTER, UNIVERSITY OF WISCONSIN, MADISON, WISCONSIN 53705 